03

\title{
Влияние коагуляции капель воды на их распределение по размерам в рабочей части аэрохолодильной установки
}

\author{
(C) М.В. Тимофреева
}

Сколковский институт науки и технологий, 143025 Сколково, Московская обл., Россия e-mail: M.Timofeeva@skoltech.ru

(Поступило в Редакцию 10 января 2018 г. В окончательной редакции 5 октября 2018 г.)

\begin{abstract}
Проведено математическое моделирование коагуляции капель конечного числа фракций размеров полидисперсной смеси, впрыскиваемых форсункой в область аэрохолодильной установки вдоль направления движения потока воздуха. Решение систем дифференциальных уравнений, описывающих изменение концентраций фракций капель, их плотностей, а также масс капель каждой из фракций с течением времени, осуществляется методом Рунге-Кутта четвертого порядка точности. Доказывается незначительность влияния тепломассобмена между веществом капель и окружающим воздухом на изменение их размеров за время движения от форсунки до рабочей части установки. Распад капель не моделируется, так как в рассмотренном режиме работы аэрохолодильного стенда значение критического числа Вебера не достигается. Результаты моделирования коагуляции капель в турбулентном потоке воздуха показывают, что распределения капель по размерам у поверхности распыляющей форсунки и в рабочей части аэрохолодильной установки не совпадают, что доказывает необходимость учета данного процесса при моделировании обледенения летательных аппаратов в наземных условиях.
\end{abstract}

DOI: $10.21883 / J T F .2019 .04 .47301 .11-18$

По статистике Международной ассоциации гражданской авиации (ИКАО) обледенение самолетов является причиной $7 \%$ всех аварий на воздушном транспорте, вызванных неблагоприятной метеорологической обстановкой. Это явление связано с наличием в атмосфере переохлажденной метастабильной воды в капельножидком состоянии при отрицательной температуре и происходит при полетах в облаках. Моделирование процесса обледенения летательных аппаратов и элементов их конструкции на стендах и установках искусственного обледенения в наземных условиях является очень важной и трудоемкой задачей, решение которой позволяет еще на стадии проектирования самолета определить влияние льда, образующегося на несущих поверхностях, на его аэродинамические характеристики, выявить степень ухудшения летно-технических характеристик (ЛТХ) и определить особенности динамики полета. Математические модели, применяемые для описания обледенения различных поверхностей летательного аппарата (ЛА), как правило, не учитывают взаимодействие облачных капель между собой [1-3]. Распределение капель по размерам в этих моделях до момента столкновения с поверхностью ЛА считается неизменным, делается предположение о потенциальности потока. Данное допущение приводит к неверному представлению о распределении капель различных фракций размеров вблизи объекта, подверженному обледенению, что может повлиять на неточности в определении формы и типа отложений на поверхности ЛА.

Определение влияния коагуляции облачных капель на характер их распределения по размерам является важной задачей. Согласно „Приложению $\mathrm{C}^{\text {‘ }}$ норм лет- ной годности самолетов транспортной категории [4], посвященному условиям обледенения, распределение облачных капель по размерам может быть представлено логарифмически-нормальным распределением со среднеарифметическим значением радиуса капель, равным $10 \mu \mathrm{m}$ (рис. 1, $a$ ).

В настоящей работе математическое моделирование коагуляции капель проводится в аэрохолодильной установке, описание которой приведено в [5]. Диаметр рас-
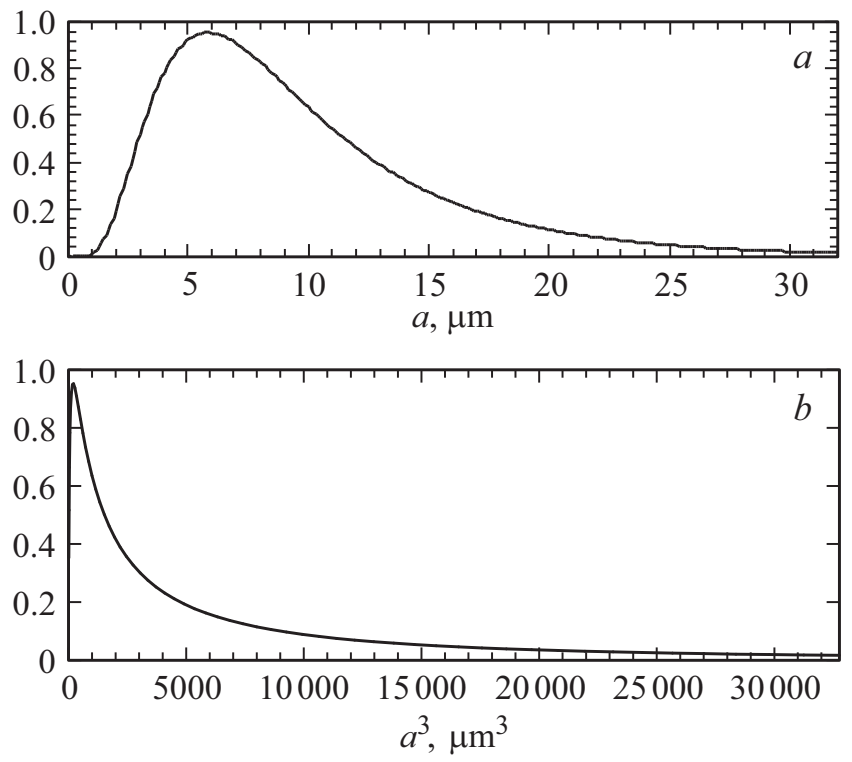

Рис. 1. $a-$ логарифмически-нормальное распределение размеров облачных капель (коэффициент дисперсии распределения равен 0.6$) ; b-$ кубы радиусов облачных капель. 
пыляющей форсунки, расположенной в тракте установки $d-2.95 \mathrm{~mm}$, расход форсунки $G-2 \mathrm{~g} / \mathrm{s}$, скорость факела воды, направленного вдоль движения потока воздуха $u_{w}-20 \mathrm{~m} / \mathrm{s}$, скорость потока воздуха в месте расположения форсунки $u_{a}-20 \mathrm{~m} / \mathrm{s}$, температура воды $T_{w}-$ $278 \mathrm{~K}$, температура воздуха $T_{a}-273 \mathrm{~K}$, значение кинетической энергии турбулентных пульсаций воздуха $k-$ $0.56 \mathrm{~J} / \mathrm{kg}$, диссипация кинетической энергии турбулентных пульсаций $\varepsilon-3.79 \mathrm{~m}^{2} / \mathrm{s}^{3}$, расстояние от места впрыска капель до рабочей части аэрохолодильной установки $x-3.2 \mathrm{~m}$. Для моделирования взаимодействия капель друг с другом рассматриваются следующие фракции капель по размерам: 2.5-3.5, 6.5-7.5, 10.5-11.5, $14.5-15.5,18.5-19.5,24.5-25.5 \mu \mathrm{m}$. Расход каждой из фракций Gi находится в соответствии с площадью под графиком (рис. $1, b)$, занимаемой каждой из групп частиц: $G_{1}=0.01 \cdot G, \quad G_{2}=0.06 \cdot G, \quad G_{3}=0.08 \cdot G$, $G_{4}=0.07 \cdot G, G_{5}=0.06 \cdot G, G_{6}=0.04 \cdot G$. Начальное значение концентраций капель выбранных фракций $n_{i}$ для диапазона $i \in[1,6]$ вычисляется из

$$
n_{i 0}=\frac{G_{i}}{V_{w i} \rho_{l} u_{w} S}
$$

где $\rho_{l}$ - плотность воды $\left[\mathrm{kg} / \mathrm{m}^{3}\right], V_{w i}$ - объем капли фракции $i\left[\mathrm{~m}^{3}\right], S$ - площадь поперечного сечения распыляющей форсунки $\left[\mathrm{m}^{2}\right]$.

Моделирование коагуляции конечного числа фракций капель полидисперсной смеси проводится путем решения дифференциальных уравнений, описывающих изменение их концентрации и плотности, а также массы капель каждой из фракций с течением времени [6]. В рассматриваемой модели коагуляции для каждой произвольной фракции с номером s все частицы меньшего размера являются „снарядами“, прилипающими к каплям данной фракции. Частицы же выделенной фракции $s-$ „снаряды“ для старших фракций. Помимо этого, учитывается уменьшение концентрации разных по размеру частиц, вследствие их взаимодействия с частицами той же фракции. Изменение концентрации частиц выбранной фракции $s$ :

$$
\begin{gathered}
\frac{\partial n_{s}}{\partial t}=-n_{s} \sum_{j=s+1}^{l} n_{j} K_{s j}-\frac{1}{2} K_{s s} n_{s}^{2}, \\
K_{s j}=\sqrt{2} V_{t} \vartheta_{s j}\left(a_{s}+a_{j}\right)^{2}, \\
K_{s s}=\sqrt{2} V_{t} \vartheta_{s s}\left(2 a_{s}\right)^{2},
\end{gathered}
$$

где $n_{s}$ - концентрация капель рассматриваемой фракции $s\left[\mathrm{~m}^{-3}\right], n_{j}-$ концентрация капель фракции $j\left[\mathrm{~m}^{-3}\right]$, $V_{t}$ - скорость турбулентных пульсаций воздуха $[\mathrm{m} / \mathrm{s}], a_{s}$, $a_{j}$ - радиусы капель рассматриваемой фракции $s[\mathrm{~m}]$ и фракции $j ; K_{s j}, K_{s s}-$ коэффициенты столкновения для случаев взаимодействия капель различных фракций и взаимодействия капель в пределах одной группы размеров.

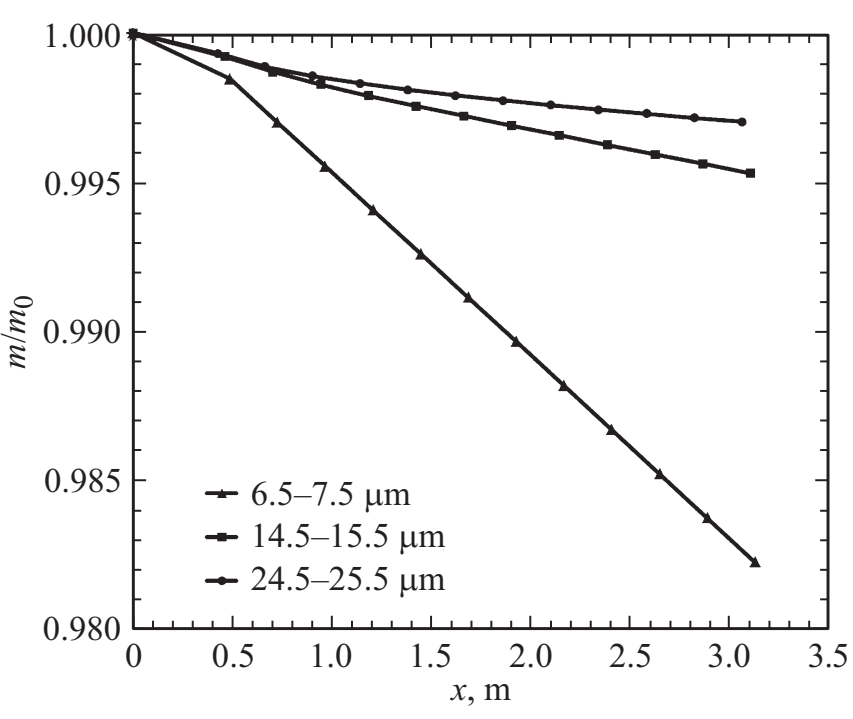

Рис. 2. Изменение масс капель, вызванное тепломассообменом с воздухом.

Важными компонентами, входящими в ядра коагуляции внутрифракционных $K_{s s}$ и межфракционных $K_{s j}$ взаимодействий капель, являются коэффициенты их столкновений $\vartheta_{s j}, \vartheta_{s s}$, характеризующие частоту взаимодействия капель, которая зависит как от размера частиц, так и от турбулентности воздуха:

$$
\begin{gathered}
\vartheta_{j s}=\sqrt{\frac{2}{3 \pi}}\left(\frac{\Omega_{s}}{1+\Omega_{s}}+\frac{\Omega_{j}}{1+\Omega_{j}}\right) \\
+\sqrt{\frac{2}{3 \pi}} \frac{\left(\Omega_{s}-\Omega_{j}\right)^{2}}{1+\left(\Omega_{s}+\Omega_{j}\right)^{2}}, \\
\Omega_{j, s}=\frac{\tau_{j, s}}{\tau^{\prime}},
\end{gathered}
$$

где $\tau^{\prime}=\frac{2 \rho_{j} a_{i}^{2}}{9 \mu}-$ время релаксации капель $[\mathbf{s}]$, $\tau^{\prime}=0.19 \frac{k}{\varepsilon}-$ время турбулентных пульсаций $[\mathrm{s}], a_{i}-$ радиус капли группы $[\mathrm{m}], \mu$ - динамическая вязкость воздуха $[\mathrm{Pa} \cdot \mathrm{s}]$. Выражение для коэффициента столкновений капель (3), согласно [6], позволяет достаточно точно отразить зависимость частоты столкновений капель от их размеров и средней скорости турбулентных пульсаций несущей среды.

Для шести выбранных фракций капель, испускаемых в воздушный поток форсункой, находящейся в тракте аэрохолодильной трубы, проводится сравнение их концентраций в момент времени $t=0$, соответствующий впрыску капель воды в поток, и в момент времени $t_{i}$ попадания капель в рабочую часть установки. Определение времени движения капель разных фракций $t_{i}$ от момента их впрыска в рабочую часть установки до момента снятия результатов проводится путем решения системы 

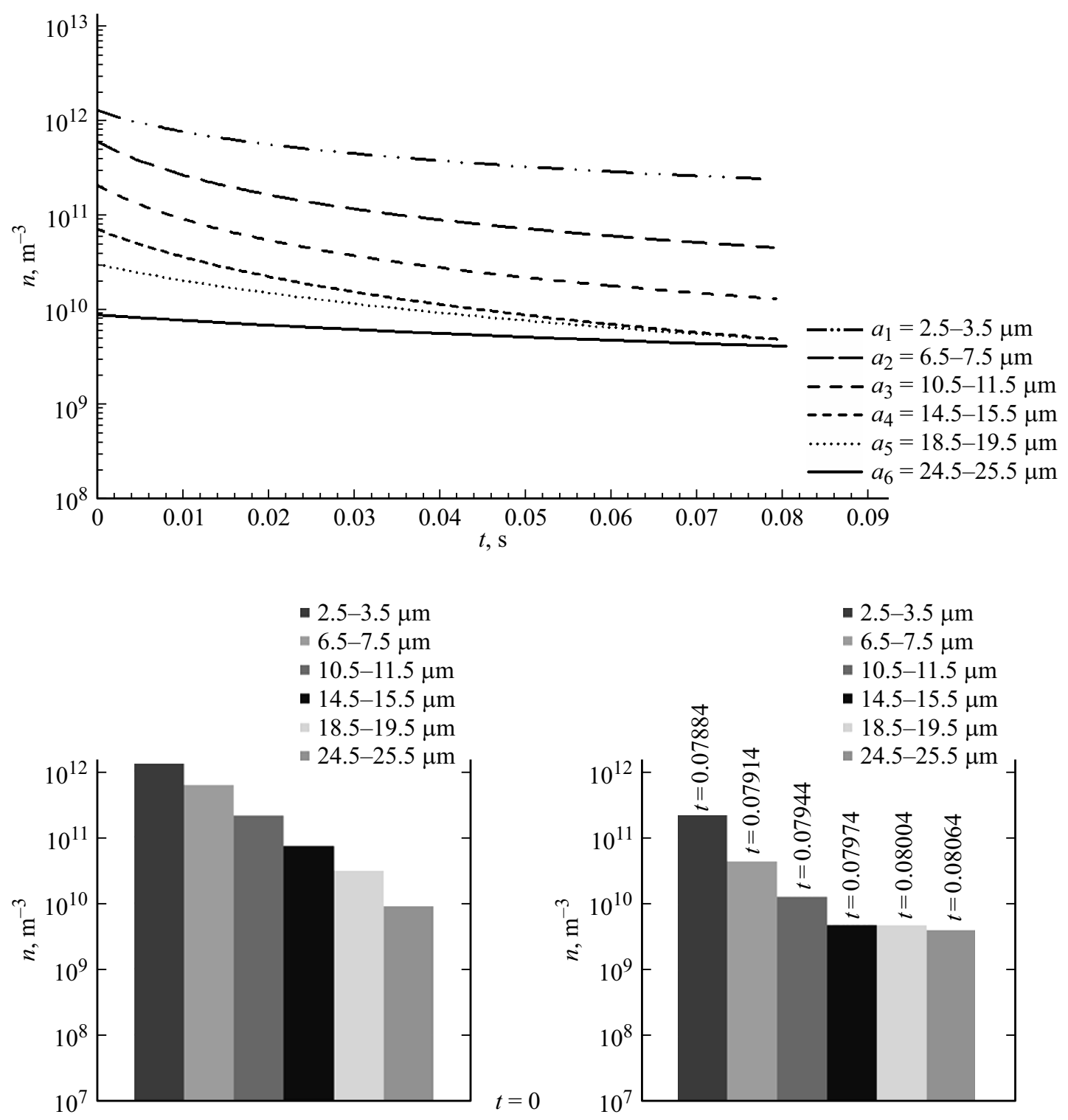

Рис. 3. Изменение концентрации капель шести фракций вдоль направления движения потока, вызванное коагуляцией.

уравнений динамики движения частицы в потоке [7]:

$$
\begin{gathered}
\frac{d \hat{u}}{d t}=\frac{3}{8} \frac{\rho_{g}}{\rho_{l}} \frac{C_{D}}{\hat{a}}\left[\left(u_{g}-\hat{u}\right)^{2}+\left(v_{g}-\hat{v}\right]^{1 / 2}\left(u_{g}-\hat{u}\right),\right. \\
\frac{d \hat{v}}{d t}=\frac{3}{8} \frac{\rho_{g}}{\rho_{l}} \frac{C_{D}}{\hat{a}}\left[\left(u_{g}-\hat{u}\right)^{2}+\left(v_{g}-\hat{v}\right]^{1 / 2}\left(u_{g}-\hat{v}\right),\right. \\
\frac{d x}{d t}=\hat{u}, \quad \frac{d y}{d t}=\hat{v},
\end{gathered}
$$

где $g$-воздух, индекс $l$ - вода, $x, y, u_{g}, v_{g}$ - система координат и соответствующие компоненты скорости несущего воздуха, $\rho-$ плотность, $\hat{u}, \hat{v}, \hat{a}-$ компоненты скорости капли и ее радиус, $C_{D}-$ коэффициент сопротивления. Система уравнений (4) решается методом Рунге-Кутта четвертого порядка точности. Время, за которое капли разных размеров проходят расстояние от форсунки до рабочей части установки $(x=3.2 \mathrm{~m})$, конечное время, используемое при решении (2).
При моделировании коагуляции пренебрегается тепломассообменом между каплями и воздухом в силу незначительности влияния данного процесса на изменение размеров капель [6]:

$$
\begin{gathered}
\frac{d \hat{a}}{d t}=-\frac{D_{v} \cdot \mathrm{Sh}}{2 \hat{a} \rho_{l}}\left[\rho_{v s}\left(T_{a}-\rho_{v \infty}\right)\right], \\
\frac{d \hat{T}}{d t}=\frac{3\left(\lambda_{g} \cdot \operatorname{Nu}\left(T_{j}-\hat{T}\right)-D_{v} L_{i v}\left(\rho_{v s}-\rho_{v \infty}\right) \cdot \mathrm{Sh}\right)}{2 \hat{a}^{2} C_{l} \rho_{l}},
\end{gathered}
$$

где индекс $a-$ поверхность капли, индекс $v-$ пар, индекс $s-$ условия насыщения, индекс $\infty-$ значения несущей среды вне потока, $\hat{T}$ - температура капли, $D_{v}$ - коэффициент молекулярной диффузии пара вещества капли, Sh - число Шервуда, $\lambda_{g}-$ коэффициент кинематической вязкости, $\mathrm{Nu}$ - число Нуссельта, $L_{l v}$ удельная теплота парообразования воды, $C_{l}$ - теплоемкость воды при нормальных условиях. 

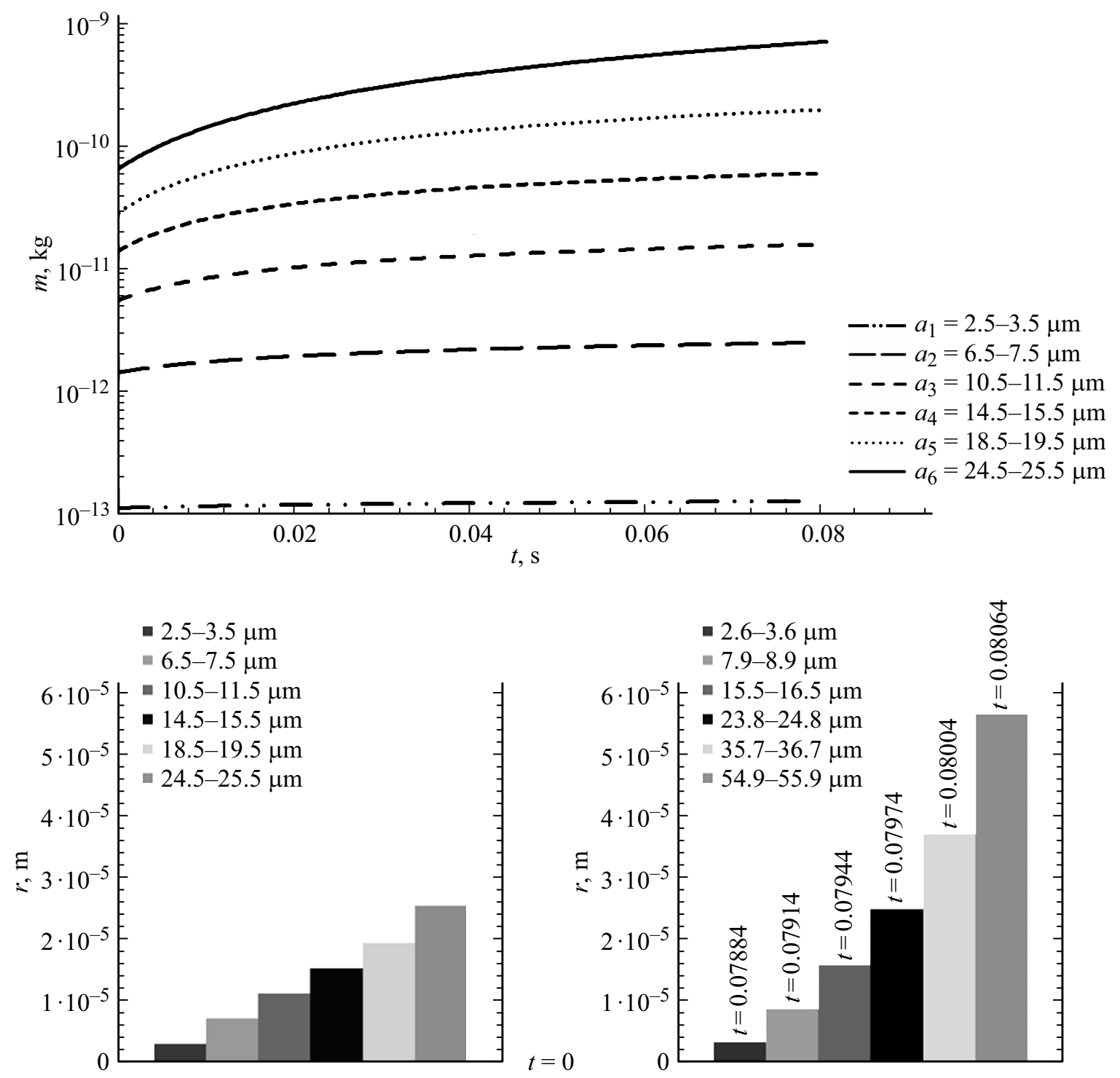

Рис. 4. Изменение массы и размера капли из каждой фракции вдоль направления движения потока.

Ниже (рис. 2) представлен результат совместного расчета (4) и (5), демонстрирующий изменение масс капель трех опорных размеров за счет их тепломассообмена с воздухом. Для наглядности результаты представлены в относительных единицах измерения.

Распад капель не моделируется в связи с тем, что число Вебера We, определяющее отношение инерции жидкости к поверхностному натяжению, не достигает своего критического значения $\mathrm{We}_{c r}=16$.

Решение системы дифференциальных уравнений, описывающих изменение со временем концентраций фракций капель разных размеров (2), проводится методом Рунге-Кутта четвертого порядка точности. Получены значения концентраций капель в разные промежутки времени их движения по тракту аэрохолодильной установки. Приведены значения концентраций капель всех рассматриваемых фракций в момент их впрыска в поток и в момент попадания капель в рабочую часть установки.
Капли разных групп размеров в разное время достигают рабочей части аэрохолодильной установки (рис. 3 ).

В процессе налипания „снарядов“ на капли больших размеров, а также из-за слипания капель внутри фракций масса частиц каждой из групп претерпевает изменения. Уравнение, описывающее изменение массы одной частицы рассматриваемой фракции $s$, выглядит следующим образом:

$$
\frac{\partial m_{s}}{\partial t}=\sum_{j=1}^{s-1} n_{j} m_{j} K_{j s}+\frac{1}{2} K_{s s} n_{s} m_{s}
$$

где $m_{s}, m_{j}-$ массы капель рассматриваемой фракции $s$ и фракции $j[\mathrm{~kg}]$.

Проводится вычисление изменения масс капель шести групп размеров, связанное с их коагуляцией. Как и в случае рассмотрения изменения концентрации капель выбранных фракций, система дифференциальных урав- 


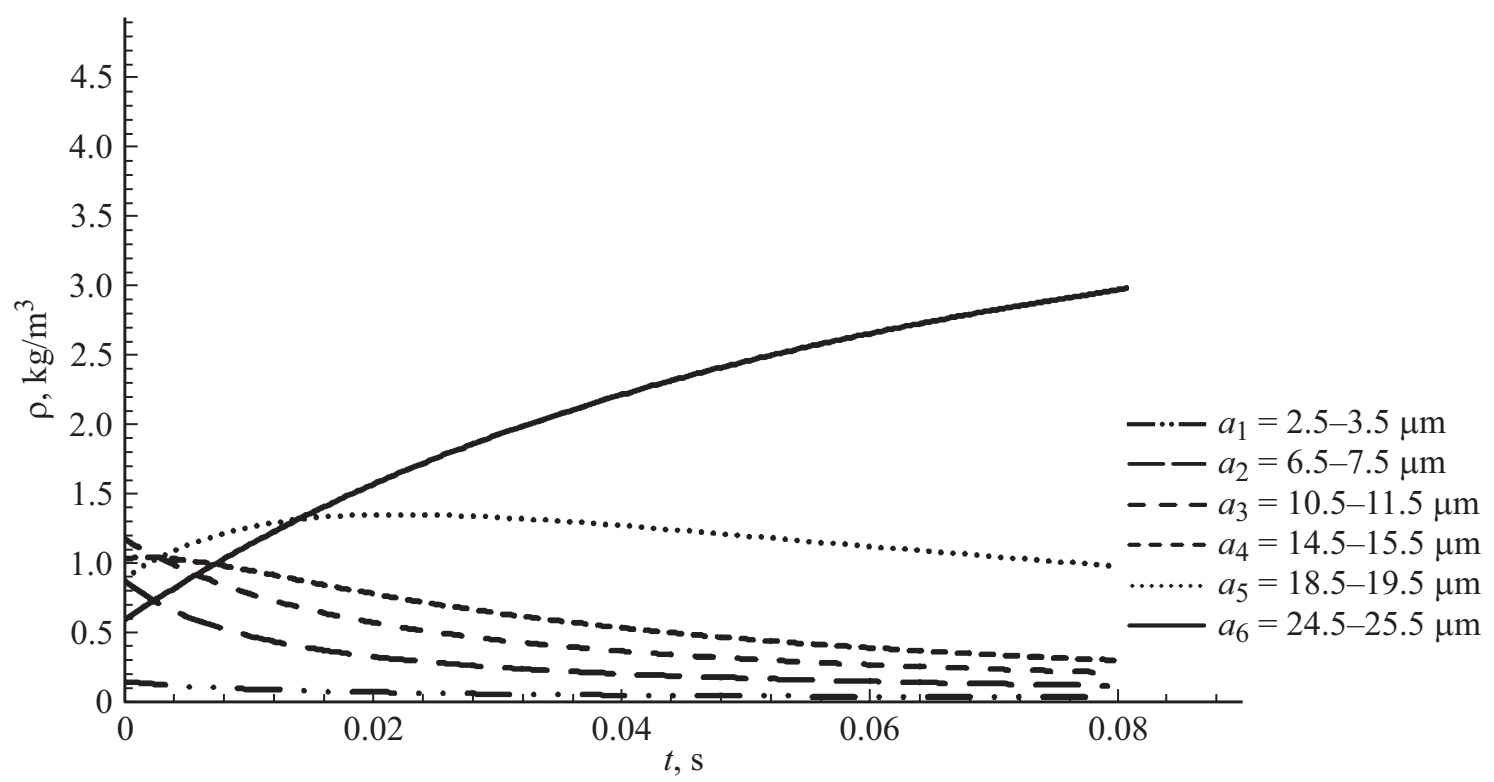

Рис. 5. Изменение плотности фракций капель вдоль направления движения потока.

нений решается методом Рунге-Кутта четвертого порядка точности. На каждом шаге вычислений учитываются значения концентраций частиц всех фракций, получаемые из системы уравнений (2). Находятся изменение масс капель вдоль направления их движения по каналу аэрохолодильной установки и значения радиусов частиц в момент времени $t=0$ и $t_{i}$ (рис. 4).

Умножив уравнение для концентрации частиц (2) на $m_{s}$, а уравнение для массы $(6)-$ на $n_{s}$ и сложив, можно получить уравнение, описывающее эволюцию плотности $s$-фракции $\rho_{s}=n_{s} m_{s}$ :

$$
\frac{\partial \rho_{s}}{\partial t}=\frac{\rho_{s}}{m_{s}} \sum_{j=1}^{s-1} K_{j s} \rho_{j}-\rho_{s} \sum_{j=s+1}^{l} K_{s j} \frac{\rho_{j}}{m_{j}}
$$

где $\rho_{s}, \rho_{j}$ - плотности капель рассматриваемой фракции $s$ и фракции $j\left[\mathrm{~kg} / \mathrm{m}^{3}\right]$, при этом $(1 \leq s \leq l)$.

Используя значения концентраций и масс капель выбранных фракций на каждом шаге по времени, можно построить изменение плотности фракций капель, вызванное их коагуляцией (рис. 5).

\section{Заключение}

Результаты, полученные при моделировании коагуляции конечного числа фракций капель полидисперсной смеси, демонстрируют, что вследствие взаимодействия капель друг с другом, большие капли стремительно увеличиваются в размерах все чаще улавливая капли меньших по размеру фракций. Поэтому концентрация мелких капель успевает значительно снизиться за время движения по каналу тракта аэрохолодильной установки, описанной в [5]. Концентрация крупных капель за время движения от места расположения распыляющей форсунки до рабочей части стенда меняется незначительно, зато их размер и массовая плотность существенно растут.

Учет влияния турбулентности воздуха на интенсивность взаимодействия капель друг с другом приводит к абсолютно иному распределению капель по размерам в сравнении с изначально заданным. В данной работе показано, что капли, достигающие рабочей части аэрохолодильной установки, значительно крупнее тех, которые моделируются при вылете из форсунки. Распределения капель по размерам у поверхности распыляющей форсунки и в рабочей части аэрохолодильной установки не совпадают. Таким образом, моделирование обледенения поверхности ЛА в наземных условиях должно осуществляться с учетом коагуляции капель, распределение по размерам которых способно значительно измениться за короткое время их движения в турбулентном потоке воздуха.

Автор выражает огромную благодарность Миллеру А.Б. и Стасенко А.Л. за ценные советы при планировании исследования и предоставление экспериментальных данных, используемых в работе.

\section{Список литературы}

[1] Ackley S.F., Templeton M.K. Computer modeling of atmospheric ice accretion. - cold regions research and engineering lab hanover nh. 1979. № crrel-79-4.

[2] Lozowski E.P., Makkonen L. Fifty Years of Progress in Modelling the Accumulation of Atmospheric Ice on Power Network Equipment // Proc. Eleventh International Workshop on Atmospheric Icing on Structures, Montreal, CD-ROM, 2005. 
[3] Dong W., Zhu J.J., Zhao Q.Y. Numerical Simulation Analysis of a Guide Vane Hot Air Anti-icing System. 2011. AIAA paper. 3944. P. 2011.

[4] Авиационные правила, Часть 25. Нормы летной годности самолетов транспортной категории // М.: Летноисследовательский институт им. М.М. Громова, 2009.

[5] Гринач Э.С., Миллер А.Б., Потапов Ю.Ф., Стасенко А.Л. Экспериментальные и теоретические исследования процессов обледенения наномодифицированных супергидрофобных и обычных поверхностей. Вестник Московского гос. обл. ун-та. Серия: Физика-математика. 2013. № 3. С. 84-92.

[6] Стасенко А.Л. Физическая механика многофазных потоков: Учебное пособие. М.: МФТИ, 2004. 136 с.

[7] Langmuir I., Blodgett K. A mathematical Investigationof Water Droplet Trajectories. U.S.A.A.F. Tech. Rep. 1946. N 5418. 47 p. 